\title{
Charging Dynamics of Aerosol OT Inverse Micelles
}

\author{
Masoumeh Karvar, ${ }^{* \dagger}{ }^{\dagger}$ Filip Strubbe, ${ }^{\dagger, \ddagger}$ Filip Beunis, ${ }^{\dagger, \ddagger}$ Roger Kemp, ${ }^{\S}$ Nathan Smith, ${ }^{\S}$ Mark Goulding, ${ }^{\S}$ \\ and Kristiaan Neyts \\ ${ }^{\dagger}$ Department of Electronics and Information Systems, Ghent University, B-9000 Ghent, Belgium \\ "Center for Nano- and Biophotonics, Ghent University, B-9000 Ghent, Belgium \\ ${ }^{\S}$ Merck Chemicals Ltd, University Parkway, Chilworth, Southampton, SO16 7QD, United Kingdom
}

ABSTRACT: Aerosol OT (AOT) is a commonly used surfactant and charging agent in nonpolar liquids. Properties such as the conductivity of AOT suspensions in nonpolar liquids and the behavior of charged $\mathrm{AOT}$ inverse micelles at interfaces have been studied recently, but still little is known about the generation dynamics of charged AOT inverse micelles. In this article, the generation dynamics of charged AOT inverse micelles in dodecane are investigated with transient current measurements. At low applied voltages, the generation rate is sufficiently fast to maintain the equilibrium concentration of charged inverse micelles, such that the current scales proportionally with the applied voltage. However, above a threshold

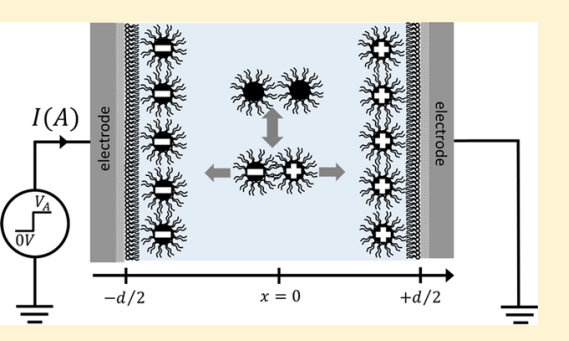
voltage the current becomes limited by the generation of charged inverse micelles. $\mathrm{Al}_{2} \mathrm{O}_{3}$.coated electrodes are used to achieve these high-voltage current measurements while reducing surface generation currents. The dependency of the resulting generation-limited currents with the micelle concentration and the liquid volume is compatible with a bulk disproportionation mechanism. The measured currents are analyzed using a model based on drift, generation, and recombination of charged inverse micelles and the corresponding generation and recombination rates of charged AOT inverse micelles have been determined.

\section{INTRODUCTION}

Surfactants are widely used for charging colloidal particles in reflective displays, ${ }^{1-8}$ inkjet printing, ${ }^{9}$ electrorheological fluids, ${ }^{10}$ emulsion polymerization, ${ }^{11,12}$ and dry cleaning. ${ }^{13}$ Over recent years, Anionic sodium bis (2-ethylhexyl) sulfosuccinate (Aerosol OT, AOT) with its double-tail and polar headgroup has attracted increasing attention in aqueous, ${ }^{14,15}$ nonaqueous ${ }^{16-19}$ and water-in-oil (w/o) microemulsions. $^{20-26}$

During the last decades, different methods have been used to investigate the properties of AOT inverse micelles in nonpolar solvents. Peri has combined ultracentrifugation, light scattering, and viscometric measurements of $\mathrm{AOT}$ in various solvents, and he has suggested that AOT inverse micelles are monodisperse and spherical. ${ }^{27}$ Ekwall et al. have found similar results by using light and X-ray scattering. ${ }^{28}$ Their studies indicate that the aggregation number $N_{\mathrm{g}}$ the number of monomers per inverse micelle, for AOT is about 20-30. Using a photon correlation experiment, Zulauf and Eicke $29,30,29,30$ have studied the aggregation number of AOT micelles over a wide range of concentrations and in different solvents. ${ }^{27,28}$ Kotlarchyk et al. have used small-angle neutron scattering (SANS) and found an aggregation number of $22 \pm 2$ monomers per micelle. ${ }^{31}$

Conductivity measurements of AOT solutions show that the conductivity scales linearly with the concentration of AOT in the range between 0.1 and $3 \mathrm{wt} \%$. Hsu et al. found that the conductivity of AOT/dodecane solutions made in a dry glovebox is about $2.4 \mathrm{nS} / \mathrm{m}$ per weight percent of AOT in dodecane. ${ }^{32}$ Karvar et al. found a conductivity of $5.3 \mathrm{nS} / \mathrm{m}$ per weight percent ${ }^{33}$ for AOT/dodecane under ambient condition.
This difference can be explained by the hygroscopic properties of AOT, which makes the micelles absorb ambient moisture, and increases the conductivity of the AOT solution. ${ }^{34}$ The charge-bearing entities are either trace ions such as formed from the dissociation of water into $\mathrm{H}^{+}$and $\mathrm{OH}^{-}$, or even charged AOT molecules that have released a $\mathrm{H}^{+}$. When two micelles collide there is a chance that these charged entities are exchanged between micelles, forming charged micelles. In the case of dissociation, a large micelle can split up in two smaller micelles which can be charged. The linear relation between conductivity and surfactant concentration can be explained by a thermodynamic mechanism of generation of charged inverse micelles, based on a disproportionation/comproportionation reaction of inverse micelles. In this disproportionation mechanism, two uncharged inverse micelles $\left(A_{0}\right)$, transfer a charge during a collision, resulting in one negatively $\left(A_{-}\right)$and one positively, $\left(A_{+}\right)$charged inverse micelle (generation) and vice versa (recombination): $:^{32,33,35}$

$$
A_{0}+A_{0} \stackrel{K}{\leftrightarrow} A_{+}+A_{-}
$$

We can assume that the valency of charged inverse micelles due to their small size can only be $z=z_{+}=-z_{-}=1$. In thermodynamic equilibrium with equilibrium constant $K$, this generation mechanism leads to $\left[A_{+}\right]\left[A_{-}\right]=K\left[A_{0}\right]^{2}$. Assuming that $\left[A_{+}\right]=\left[A_{-}\right]$to keep overall charge neutrality, we find indeed $n_{ \pm}=\left[A_{ \pm}\right]=\sqrt{ } K\left[A_{0}\right]=\sqrt{ } K n_{0}$ in which $n_{0}, n_{+}$, and $n_{-}$

Received: May 7, 2015

Revised: August 12, 2015

Published: September 16, 2015 
are, respectively, the concentrations of uncharged, positively and negatively charged inverse micelles. The generation rate of the disproportionation of charged inverse micelles is $\beta n_{0}^{2}$ with $\beta$ $\left(\mathrm{m}^{3} / \mathrm{s}\right)$ the generation rate constant. The reverse process has a rate $\alpha n_{+} n_{-}$, with $\alpha\left(\mathrm{m}^{3} / \mathrm{s}\right)$ the recombination rate constant. Since the conductivity is the product of the concentration of charges and their mobilities, different methods are often used to estimate these properties separately.

One option is to use measured values of the radius of charged inverse micelles and Stokes' law to calculate the electrophoretic mobility such that the concentration of charged inverse micelles can be estimated. Furthermore, the total number of inverse micelles can be estimated from the known concentration of surfactant molecules and the aggregation number. In this way, the ratio of charged to uncharged inverse micelles can be extracted. For example, using the hydrodynamic radius $R=1.6 \mathrm{~nm}$ of inverse AOT micelles reported in literature ${ }^{29-32}$ and the viscosity of dodecane $\left(\eta=1.38 \times 10^{-3}\right.$ $\left.\mathrm{kg} \cdot \mathrm{m}^{-1} \cdot \mathrm{s}^{-1}\right)$ the mobility of charged AOT inverse micelles with unit charge becomes $\mu=\mu_{+}=-\mu_{-}=e /(6 \pi \eta R)=3.8 \times 10^{-9}$ $\mathrm{m}^{2} \mathrm{~V}^{-1} \mathrm{~s}^{-1}$. Then, a conductivity of $5.3 \mathrm{nS} / \mathrm{m}$ per weight percent corresponds to a concentration of charged inverse micelles $\bar{n}=$ $\sigma /(2 e \mu)=n_{+}=n_{-}=4.3 \times 10^{18} \mathrm{~m}^{-3}$ per weight percent of AOT. Assuming that all surfactant molecules form inverse micelles and that all the inverse micelles have the same aggregation number $N_{\mathrm{g}}=30$, we can estimate the total concentration of inverse micelles as $\bar{n}_{\text {tot }}=\left(\rho \mathrm{m} / \mathrm{m}_{\text {mol }} N_{\mathrm{g}}\right) c$, where $m_{\mathrm{mol}}$ is the mass of one AOT surfactant molecule $\left(7.38 \times 10^{-25}\right.$ $\mathrm{kg}$ ), and $c$ is the weight fraction of AOT in the solution. Using the value of $\rho_{\mathrm{m}}=1100 \mathrm{~kg} / \mathrm{m}^{3}$ for the density of an AOT inverse micelle we find $\bar{n}_{\text {tot }}=4.9 \times 10^{23} \mathrm{~m}^{-3}$ per weight percent of AOT. From the estimated values of $\bar{n}_{\text {tot }}$ and $\bar{n}$ the equilibrium constant for generation/recombination of inverse micelles can be calculated as $K=\bar{n}^{2} / \bar{n}_{0}^{2}=\bar{n}^{2} /\left(\bar{n}_{\text {tot }}-2 \bar{n}\right)^{2}$ which yields $7.7 \times$ $10^{-11}$. The fraction of charged versus uncharged inverse micelles $\chi=\left(n_{+}+n_{-}\right) / n_{0}$ is then $1.7 \times 10^{-5}$. $^{32}$ This indicates that the majority of the inverse micelles are neutral, as expected from the strong electrostatic interactions in nonpolar liquids.

Thermodynamic models are used in the second way to study the equilibrium properties of charged inverse micelles. ${ }^{32,35,36}$ In these models the Bjerrum length $\lambda_{B}=e^{2} /\left(4 \pi \epsilon_{0} \epsilon_{r} k_{B} T\right)$ is the characteristic separation between two ions at which their Coulombic attraction is exactly balanced by the thermal energy, with $\epsilon_{0}$ the vacuum permittivity, $\epsilon_{r}$ the relative dielectric constant of the liquid, $k_{\mathrm{B}}$ Boltzmann's constant and $T$ the absolute temperature. For dodecane $\lambda_{B}$ is $28 \mathrm{~nm}$, and the electrostatic energy of an inverse micelle of radius $R$, carrying a charge ze divided by $k_{B} T$ is $U_{\mathrm{el}}(z) / k_{\mathrm{B}} T=z^{2} \lambda_{\mathrm{B}} / 2 R^{35,37}$ In thermodynamic equilibrium, the reaction constant $K$ is related to the difference in Gibbs free energy between the left and right side of the disproportionation/comproportionation reaction eq 1 and this difference can be approximated by the electrostatic energy of the two charged inverse micelles. Considering the formulas for $K$ and $\chi$ we see that they are related by $\chi=2 \sqrt{ } K$. Then, the fraction of charged micelles $\chi$ with Boltzmann statistics can be written as

$$
\chi=2 \exp \left(U_{e l}(z) / k_{\mathrm{B}} T\right)=2 \exp \left(z^{2} \lambda_{B} / 2 R\right)
$$

Again with the example of $R=1.6 \mathrm{~nm}$ we find $\chi=1.5 \times$ $10^{-5,35}$ which is in good agreement with the value reported from the first method.

Third, transient current measurements are an interesting tool to study the equilibrium properties of inverse micelles, Here, if the electric field is sufficiently high and the generation sufficiently low that the transient current due to the initially present charges can be observed, theory can be used to extract the concentration and mobility of the charges separately. This method has been successfully used to study inverse micelles of PIBS (OLOA1200), ${ }^{38,39}$ OLOA 11k, ${ }^{40}$ Solsperse 13940, ${ }^{40}$ Span $80,{ }^{40}{\text { Span } 85^{40} \text { and AOT. }}^{7,33,40}$ Detailed theoretical and experimental investigations have been carried out to map the rich electrodynamics of charged inverse micelles during these current transients depending on the charge content and applied voltage. ${ }^{41-43}$ But it is also found that the behavior of charged inverse micelles at electrode interfaces can be different depending on the type of micelle. ${ }^{33}$ Whereas charged inverse micelles from the surfactant OLOA $11 \mathrm{~K}$ form a diffuse double layer at the electrodes leading to specific transient currents, most of the charged AOT inverse micelles stick to the electrodes in a thin layer resulting in different transient currents. ${ }^{33}$ For AOT, we observed that at voltages below $10 \mathrm{~V}$ the measured current upon application of a voltage step $(0 \rightarrow$ $\left.V_{\mathrm{A}}\right)$ is exponentially decaying with a time constant that is independent of the applied voltage and proportional to the AOT concentration and the device thickness. This behavior can be explained by the high generation rate of AOT inverse micelles giving the suspension resistor-like properties, and the charging of an interface capacitance causing exponential screening of the electric field.

Besides studying equilibrium properties such as the conductivity, concentrations of uncharged and charged inverse micelles, the mobility of charged inverse micelles and micelle interactions at the electrodes, transient current measurements have been used to investigate the dynamics of the generation of charged inverse micelles. For the surfactant OLOA1200 ${ }^{38}$ and in more detail for OLOA $11 \mathrm{~K}^{40}$ this is achieved by measuring generation-limited quasi steady-state currents. These generation-limited currents are due to bulk disproportionation since they scale with the square of the surfactant concentration and scale proportionally with the liquid volume. The generation and recombination constants have been determined. However, for AOT this approach is much more challenging because the generation rate is much higher. Much higher electric fields are then required to be able to measure the generation-limited current.In this paper, we use transient current measurements on AOT/dodecane solutions at relatively high electric fields, which allow to identify the dynamic properties of AOT inverse micelles and to obtain the generation and recombination rate constants. Measurements are performed over a range of applied voltages, AOT concentrations, and cell thicknesses. Experiments with and without $\mathrm{Al}_{2} \mathrm{O}_{3}$ charge blocking layers are carried out, to distinguish between faradaic and nonfaradaic processes. We present a simple analytical model based on drift and generation/recombination to describe the generationlimited steady-state current.

\section{MATERIALS AND METHODS}

Transient current measurements are carried out to investigate the properties of inverse micelles in AOT/dodecane mixtures. Devices are fabricated using two glass plates with indium-tinoxide (ITO) electrodes with overlapping area $S=1 \mathrm{~cm}^{2}$. The thickness $d$ of the devices (i.e., the distance between the electrodes) is controlled by glass spacer beads in the UV curing glue (Norland) at the edges of the device. Devices with thicknesses $d=10,27$, and $57 \mu \mathrm{m}$ are made, and the thickness is verified measuring optical interference with a PerkinElmer 
Lambda 35 spectrometer. Before each measurement the volume between the electrodes is filled with a mixture of high-purity $(99.9 \%) n$-dodecane (dielectric constant $\varepsilon_{\mathrm{r}}=2$ ) and surfactant AOT (Sigma-Aldrich) with mass fractions $\phi_{\mathrm{m}}=$ $0.0003,0.001,0.003,0.01$, or 0.03 (corresponding to $0.5,1.7$, $5.1,17$, or $51 \mathrm{mM}$ ). AOT micelles are extraordinary hydroscopic, and water solubility is never zero even in apolar solvents, although less than in the polar solvents. Eicke et al. have studied the properties and solubilization capacities of micelles in water-oil media, ${ }^{25}$ and they proposed a model in order to predict the electrical conductivity of AOT-water-apolar microemulsions. ${ }^{26}$ In the present study, no measures were taken to eliminate traces of ambient humidity in our mixtures. From exposure of a mixture to the ambient the conductivity can eventually increase up to $50 \%$ when the inverse micelles become saturated with water corresponding to about $w=$ $\left[\mathrm{H}_{2} \mathrm{O}\right] /[\mathrm{AOT}]=0.25$, but without changing their physical behavior. ${ }^{33}$ Here, to minimize ambient water absorption, our measurements have been carried out immediately after preparation.

The schematic structure of device is shown in Figure 1. The electric current upon application of a DC voltage step $\left(0 \rightarrow V_{\mathrm{A}}\right.$

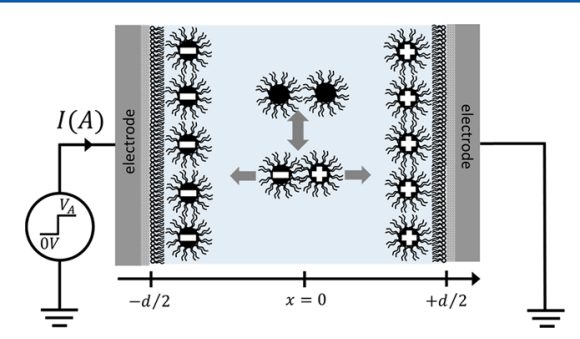

Figure 1. Schematic representation of the measurement devices with thickness $d$, with or without $\mathrm{Al}_{2} \mathrm{O}_{3}$ coating on the ITO electrodes. The disproportionation generation of charges in the bulk as well as the formation of a charged Helmholtz layer are indicated. The transient current $I$ is measured upon application of a step voltage $0 V$ to $V_{\mathrm{A}}$.

with $V_{\mathrm{A}}$ from $0.01 \mathrm{~V}$ up to $50 \mathrm{~V}$ ) is measured with a setup based on a Keithley 428 current amplifier. Before each voltage step the device is short-circuited for several hours to reach the same initial equilibrium condition. Similar measurements have been carried out with devices in which the ITO electrodes have been coated by $50 \mathrm{~nm} \mathrm{Al}_{2} \mathrm{O}_{3}$ by e-beam evaporation. With this additional layer, faradaic currents, and currents related to surface generation of charged inverse micelles are reduced. We have observed that the deposition method for the $\mathrm{Al}_{2} \mathrm{O}_{3}$ coating can have a strong influence on the surface generation properties. In the experiments we used $\mathrm{Al}_{2} \mathrm{O}_{3}$ coated electrodes that give the lowest surface generation.

\section{EXPERIMENTAL DATA}

From previous studies on AOT we know that for sufficiently low voltages $\left(V_{\mathrm{A}}<1 \mathrm{~V}\right)$, the measured current decreases exponentially over time. ${ }^{33}$ The initial current at $t=0$ is proportional to $V_{\mathrm{A}}$ and to the mass fraction of surfactant $\phi_{\mathrm{m}}$, but is inversely proportional to the thickness $d$. The time constant of the exponential decay $\tau$ is proportional to the thickness $d$ and inversely proportional to the mass fraction of surfactant $\phi_{\mathrm{m}}$. These dependencies are explained by assuming first that the generation of charged inverse micelles in the bulk is sufficiently fast to keep the concentrations of positively and negatively charged inverse micelles in the bulk at a constant equilibrium level, and second that most of the positively and negatively charged inverse micelles form a thin charged Helmholtz layers of about nanometers when they reach the electrode interfaces. Therefore, the AOT suspension behaves as a resistor which charges up two interface capacitance, resulting in the observed exponential decay.

For sufficiently high voltages (typically $V_{\mathrm{A}}>1 \mathrm{~V}$ ) the current drops with a factor 2 to 10 during the first few milliseconds, to a value which we will refer to as the steady-state current. Figure 2 shows an example

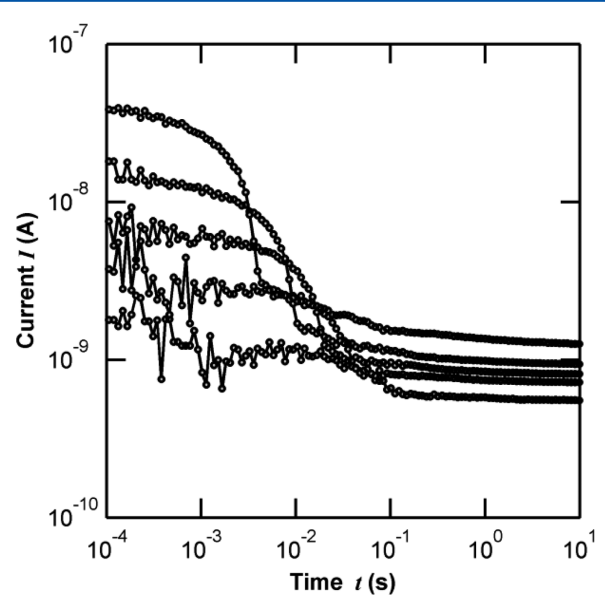

Figure 2. Transient current measurements for a mixture with 0.0003 mass fraction of AOT in dodecane, thickness $d=27 \mu \mathrm{m}$, electrode area $S=1 \mathrm{~cm}^{2}$, with $\mathrm{Al}_{2} \mathrm{O}_{3}$ coating, for applied voltages $V_{\mathrm{A}}=2,5,10,20$, and $50 \mathrm{~V}$.

of this situation measured with a cell with $d=27 \mu \mathrm{m}$, with a $50 \mathrm{~nm}$ thin $\mathrm{Al}_{2} \mathrm{O}_{3}$ coating on the ITO electrodes, and filled with a suspension with mass fraction 0.0003 of AOT in dodecane for voltage steps with $V_{\mathrm{A}}=2,5,10,20$, and $50 \mathrm{~V}$. In the experiments values of the steadystate current are taken at $100 \mathrm{~ms}$ after application of the voltage step. These transient currents can be explained as follows. Because at these voltages the electric field is relatively high, the assumption that the generation rate is fast enough to keep the concentration of charged inverse micelles constant is no longer valid. Therefore, the drop in the current during the first (tens of) milliseconds corresponds to the arrival of the initially present charged inverse micelles at the electrodes. After the initially present micelles have arrived near the electrodes, the remaining current is due to the generation and motion of newly generated charged inverse micelles toward the electrodes. Because the generation rate of new charged inverse micelles is not sufficient to keep up with the removal of charged inverse micelles from the bulk to the electrodes, the concentration of positively and negatively charged inverse micelles in the bulk is lower than in equilibrium and the steady state current is lower than the initial current. As expected, when the voltage is increased the initial current increases and in the first $10 \mathrm{~ms}$ the current drops over the electrodes. The transient time of charged inverse micelles is linearly proportional to the applied voltage and inversely proportional to the thickness of the device $d$. The steadystate current increases slightly with the voltage, about a factor 2 when the voltage is increased with a factor 25 , confirming that the steadystate current is limited by generation and not by the electric field.

In a previous study, we showed that using $\mathrm{Al}_{2} \mathrm{O}_{3}$ blocks faradic reactions and minimizes the steady state current. Using this method, the initial transient current can be more easily observed in devices with a layer of $\mathrm{Al}_{2} \mathrm{O}_{3}$ on top of the ITO and it is possible to extract the mobility and concentration of charged inverse micelles of AOT. In our previous study we found a mobility of $3.6 \times 10^{-9} \mathrm{~m}^{2} \mathrm{~V}^{-1} \mathrm{~s}^{-1}$, and a concentration of $6.0 \times 10^{18} \mathrm{~m}^{-3}$ per weight percent for charged AOT inverse micelles. ${ }^{40}$

The steady state currents for devices with $\mathrm{Al}_{2} \mathrm{O}_{3}$ coated electrodes with thicknesses of 10, 27, and $57 \mu \mathrm{m}$ are shown in Figures $3(\mathrm{a}-\mathrm{c})$ as a function of the applied voltage $V_{\mathrm{A}}$. For low AOT concentrations and thin cells saturation of the current is observed above a certain threshold voltage, while for high AOT concentrations and thick cells a continuous linear relation with the voltage is observed. 

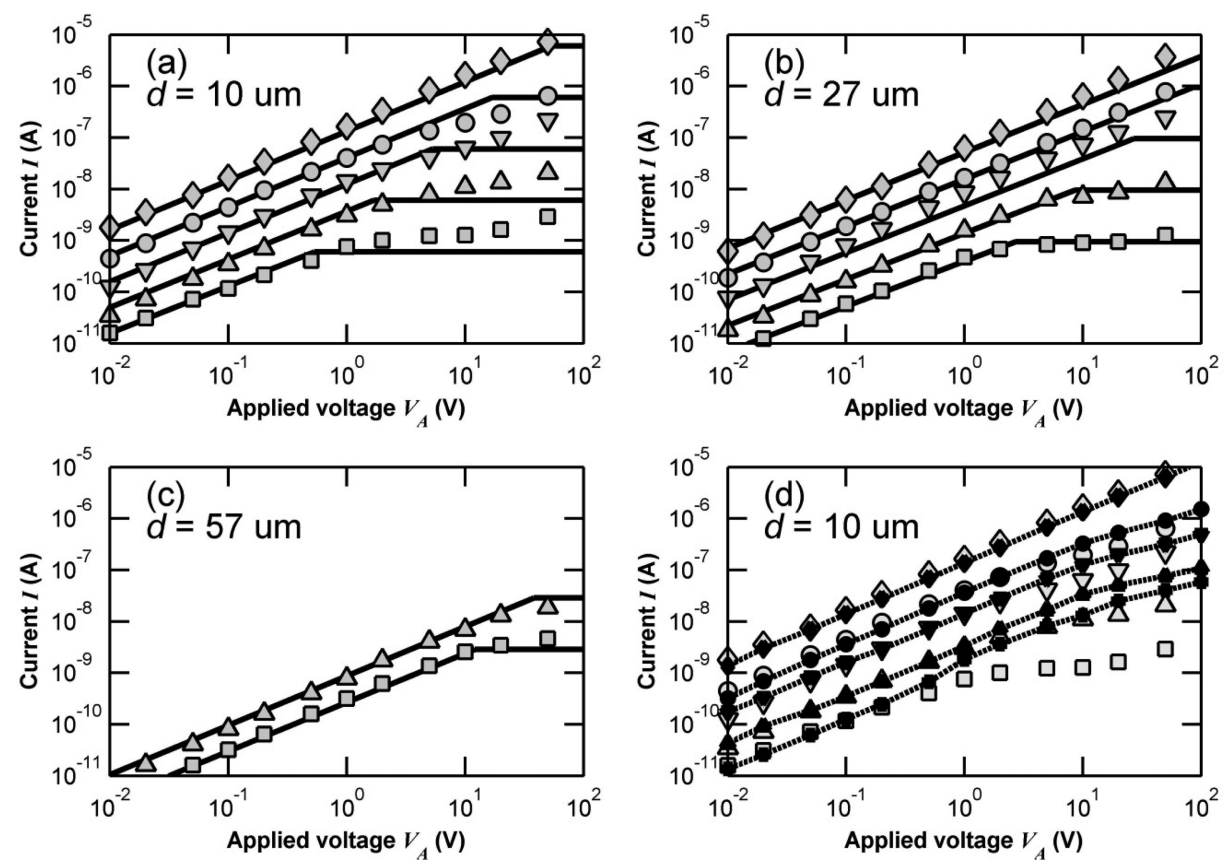

Figure 3. Voltage dependency of measured steady state currents (symbols) for devices with (a) $d=10 \mu \mathrm{m}$, (b) $d=27 \mu \mathrm{m}$, and (c) $d=57 \mu \mathrm{m}$, with surface area of the electrodes $S=1 \mathrm{~cm}^{2}$ and with $\mathrm{Al}_{2} \mathrm{O}_{3}$ coated electrodes. The solid lines show the analytical model. The mass fraction of AOT in dodecane $\phi_{\mathrm{m}}$ is presented as $0.0003(\boldsymbol{\nabla}), 0.001(\boldsymbol{\Delta}), 0.003(\boldsymbol{\nabla}), 0.01(\boldsymbol{\bullet})$, and $0.03(\boldsymbol{\nabla})$. (d) Comparison of the steady state currents in devices with (white symbols) and without (black symbols) $\mathrm{Al}_{2} \mathrm{O}_{3}$ coating on the electrodes for the device with $d=10 \mu \mathrm{m}$.

For example, in Figure $3 \mathrm{a}$ the measurement in a $10 \mu \mathrm{m}$ device at the lowest concentration of $\phi_{\mathrm{m}}=0.0003$ shows a linearly increasing current up to the saturation voltage of $1 \mathrm{~V}$. Above this saturation voltage the current is not exactly constant but is slowly increasing less than proportionally with the voltage. Because of this it makes sense to define the saturation voltage as the voltage at which a significant deviation from the linear increase is observed. Interestingly, for increasing AOT concentrations the saturation voltage increases as well, respectively $V_{\text {sat }}=1,2,6,10$ for $\phi_{\mathrm{m}}=0.0003,0.001,0.003$, and 0.01 . For the highest concentration $\phi_{\mathrm{m}}=0.03$ no saturation is observed, and we expect that saturation occurs above $50 \mathrm{~V}$. In Figure $3 \mathrm{~b}$ similar observations are made but for the same AOT concentrations the saturation voltage is systematically higher. Here we find $V_{\text {sat }}=3,10$, and $50 \mathrm{~V}$ respectively for $\phi_{\mathrm{m}}=0.0003,0.001,0.003$, while for the two highest concentrations a linear increase is observed all the way up to $50 \mathrm{~V}$. Note that since the distance between the electrodes is larger, the currents at similar applied voltages are lower than in Figure 3a. In Figure $3 \mathrm{c}$ at the same concentrations the saturation voltage is at even larger values: $V_{\text {sat }}=20$ and $50 \mathrm{~V}$ respectively for $\phi_{\mathrm{m}}=0.0003$ and 0.001 . Figure $3 \mathrm{~d}$ compares the voltage dependency of the measured steady state currents for devices with and without $\mathrm{Al}_{2} \mathrm{O}_{3}$ blocking layer, with $d=10 \mu \mathrm{m}$ and $\phi_{\mathrm{m}}=0.0003,0.001,0.003,0.01$, and 0.03 . While saturation of the current is observed for the measurements with $\mathrm{Al}_{2} \mathrm{O}_{3}$ coated electrodes for the lower concentrations, this is not so clear for plain ITO electrodes where in some cases the current even appears to increase more than linearly. Therefore, since we are primarily interested in generation-limited currents in the bulk and want to avoid other generation mechanisms, we will in the following use only the measurements for devices with $\mathrm{Al}_{2} \mathrm{O}_{3}$ coated electrodes.

In general it appears that at low voltages the current is linear with the applied voltage, with AOT concentration and inversely proportional to device thickness. The saturation voltage $V_{\text {sat }}$ appears to increase proportionally with the AOT concentration and with the device thickness. As a result the generation-limited current $I_{\text {gen }}$ has approximately a quadratic dependency on the AOT concentration and a linear dependency on the device thickness. In the next section, we show that this trend can be explained with the bulk disproportionation generation-limited mechanism.

\section{ANALYTICAL MODEL}

Inverse micelle dynamics in a AOT/dodecane mixtures are governed by generation and recombination of charged inverse micelles, diffusion, drift of charged inverse micelles in an electric field, and electro- or physicochemical reactions at the electrodes which are minimized by using $\mathrm{Al}_{2} \mathrm{O}_{3}$ coated electrodes. Based on the observation that the conductivity of AOT suspensions is proportional with the surfactant concentration, ${ }^{32,33,35}$ and on the scaling of the generationlimited currents with voltage, AOT concentration and device thickness in Figure 3, we assume that charged inverse micelles in AOT/dodecane mixtures are generated by a bulk disproportionation mechanism ${ }^{33}$ as indicated in eq 1 . Assuming that there are no faradaic processes occurring at the electrode interfaces, which is a reasonable approximation when $\mathrm{Al}_{2} \mathrm{O}_{3}$ coated electrodes are used, the transport equation of charged inverse micelles in a one-dimensional device can be written as

$$
\frac{\partial n_{ \pm}}{\partial t}=D \frac{\partial^{2} n_{ \pm}}{\partial x^{2}}-\frac{\partial}{\partial x}\left(n_{ \pm} \mu_{ \pm} E\right)+\beta n_{0}^{2}-\alpha n_{+} n_{-}
$$

where the position- and time-dependent concentrations of uncharged, positively and negatively charged inverse micelles are denoted as $n_{0}, n_{+}$, and $n_{-}\left(\mathrm{m}^{-3}\right)$ and the valency of the charged inverse micelles is assumed to be unity $z=1$. $^{41,42} E$ is the position- and time-dependent electrical field $(\mathrm{V} / \mathrm{m}), D$ is the diffusion coefficient $\left(\mathrm{m}^{2} / \mathrm{s}\right)$ which is related to the mobility of charged inverse micelles $\mu$ by $D=\mu k_{\mathrm{B}} T / z e$. The electric field is determined by the externally applied potential difference $V_{\mathrm{A}}$ and by space charge present in the bulk through Gauss's law $\epsilon_{\mathrm{r}} \epsilon_{0}(\partial E / \partial x)=\rho$ where $\rho$ is the space charge density conserving overall charge neutrality $\int_{-d / 2}^{d / 2} \rho d x=0$. The generation and recombination rates are respectively $\beta n_{0}^{2}$ and $\alpha n_{+} n_{-}$. No electric field dependency of $\alpha$ and $\beta$ is considered in this work. In the thermal equilibrium before a voltage is applied we assume that 
the charge distributions are homogeneous such that eq 3) leads to

$$
\bar{n}=n_{t_{t=0}}=n_{-_{t=0}}=\sqrt{\frac{\beta}{\alpha}} \bar{n}_{0}
$$

As explained before, the dynamics of the charged inverse micelles can be divided into two phases after a voltage is applied across the electrodes. In the first phase, all initially present charged inverse micelles move toward the electrodes of the opposite polarity. The second phase starts when all initially present charges have arrived and only newly generated charged inverse micelles contribute to the current.

In our measurements the applied voltage ranges from $10 \mathrm{mV}$ to $50 \mathrm{~V}$. But in the most relevant cases where the saturation and generation-limited currents are observed, the applied voltages are much higher than the thermal voltage, $V_{T}=k_{\mathrm{B}} T / e=25 \mathrm{mV}$, so that diffusion can be ignored. In the steady state situation at voltage $V_{\mathrm{A}}$ any time variation can be neglected and eq 3 reduces to

$$
\frac{\partial}{\partial x}\left(n_{ \pm} \mu_{ \pm} E\right)-\beta n_{0}^{2}+\alpha n_{+} n_{-}=0
$$

Now we can consider two cases for the applied voltage with respect to the saturation value of the voltage $V_{\text {sat }}\left(\phi_{\mathrm{m}}, d\right)$ which depends of $\phi_{\mathrm{m}}$ and $d$. For voltages $V$ much lower than $V_{\text {sat }}\left(\phi_{\mathrm{m}}\right.$ d) the first term due to drift can be neglected compared to the generation and recombination terms, such that the solution of eq 5 becomes $\bar{n}_{t=0}=n_{+}(t=0)=n_{-}(t=0)=(\beta / \alpha)^{1 / 2} \bar{n}_{0}$ From the current density $J=2 e n \bar{\mu} E$ from the contributions of both positively and negatively charged inverse micelles, the electric current can then easily be calculated:

$$
I_{\text {lin }}=\frac{\sigma V S}{d}=\frac{2 e \mu \bar{n} V S}{d}
$$

When the applied voltage is much larger than the saturation voltage, the drift term in eq 5 cannot be ignored anymore. From earlier studies on AOT inverse micelles in dodecane we know that when a voltage is applied, there is no sign of screening of the electric field in the bulk related to a Debye double layer capacitance. ${ }^{33}$ Instead, we observed that, apart from the initial transient due to the initially present charges, the measured currents upon application of a voltage step are exponentially decaying, with a time constant which does not depend on the applied voltage, but which is proportional to the thickness of the device and inversely proportional to the mass fraction of AOT surfactant. The exponential decay of the transient currents and the dependencies are consistent with an equivalent RC circuit model. Physically, this behavior is interpreted, first by the high generation rate of charged inverse micelles giving the suspension resistor like properties, and second by the buildup of charged inverse micelles in thin interface layers at both electrodes, acting as capacitors. As a result, the electric field in the bulk is screened by the charging of these interface capacitances with a time constant that depends on the properties of the device (typically a few to hundreds of seconds). However, on the time scale of the measurements in the present study (few hundred milliseconds), the influence of charge in these interface layers can be ignored, and in good approximation we can consider that the electric field in the bulk remains equal to its initial value $V / d$. For this reason, we can assume that the electric field in the bulk remains homogeneous even after large amounts of charged micelles have accumulated at the electrodes. Due to the strong electric field, charged inverse micelles are drifting faster to the electrodes than they are generated and the average concentration of charged inverse micelles in the bulk decreases. In the limit of high voltages under consideration here, the concentration of charges is so low that the recombination term $\alpha n_{+} n_{-}$in eq 5 can be neglected with respect to the generation term $\beta n_{0}^{2}$. This generation term $\beta n_{0}^{2}$ is approximately constant since the concentration of uncharged inverse micelles practically does not decrease on the time scale of the experiment Assuming with the above arguments that $n_{0}=\bar{n}_{0}$ and $E=V_{\mathrm{A}} / d$ in the bulk, and using $\mathrm{Al}_{2} \mathrm{O}_{3}$ coated electrodes as a blocking boundary condition, simply no charges are generated at the electrodes and the flux of generated charged inverse micelles is zero at the electrodes with the same polarity. Due to the same blocking boundary condition, the newly generated charged inverse micelles accumulate at the oppositely polarized electrodes. With this blocking boundary condition at the electrodes eq 5 can be solved:

$$
n_{ \pm}=\left(x \pm \frac{d}{2}\right) \frac{\beta n_{0}^{2}}{\mu_{ \pm} E}
$$

The result is a linearly increasing concentration profile for $n_{+}$ and $n_{-}$, with a zero concentration at the electrode with the same polarity. In this case the steady state current becomes independent of the applied voltage:

$$
I_{\text {gen }}=e d \beta n_{0}^{2} S=e d \alpha \bar{n}^{2} S
$$

As can be seen from eqs 6 and 8 , the steady state current increases linearly with the applied voltage until it saturates to a constant value $I_{\text {gen }}$ at sufficiently high voltages. $I_{\text {gen }}$ is proportional with the device thickness and scales quadratically with the AOT concentration. A formula for the saturation voltage $V_{\text {sat }}$ above which the current becomes generationlimited, can then be found from the intersection between the two asymptotically linear approximations:

$$
V_{\text {sat }}=\frac{d^{2} \alpha \bar{n}}{\mu}=\frac{d^{2} \alpha \sigma}{2 e \mu^{2}}
$$

From eq 9 it appears that the saturation voltage is proportional to the AOT concentration and to the square of the device thickness $d$.

\section{QUANTITATIVE STUDY}

In this section, we calculate the generation rate of charged AOT inverse micelles by comparing the experiments to the analytical model. First, for all the measurements below the saturation voltage, we find an excellent fit using $I=\sigma V S / d$ eq 6 with conductivity $\sigma=6 \mathrm{nS} / \mathrm{m}$ per weight percent of AOT. From a detailed analysis of transient currents in devices with $\mathrm{Al}_{2} \mathrm{O}_{3}-$ coated electrodes ${ }^{40}$ similar as shown in Figure 2 this conductivity corresponds to the mobility $\mu=3.6 \times 10^{-9}$ $\mathrm{m}^{2} \mathrm{~V}^{-1} \mathrm{~s}^{-1}$, and the concentration of charged AOT inverse micelles $\bar{n}=4.95 \times 10^{18} \mathrm{~m}^{-3}$ per weight percent.

Second, at voltages above the saturation voltage the generation rate $\beta n_{0}^{2}=2.6 \times 10^{21} \mathrm{~m}^{-3} \mathrm{~s}^{-1}$ at $1 \mathrm{wt} \%$ of AOT is estimated be fitting eq 8 to all measured generation-limited currents. Since in equilibrium $\beta n_{0}^{2}=\alpha \bar{n}^{2}$, and since $\bar{n}=4.95 \times$ $10^{18} \mathrm{~m}^{-3}$ per weight percent for charged AOT inverse micelles, the best fitting value for the recombination constant is $\alpha=9.0$ $\times 10^{-17} \mathrm{~m}^{3} / \mathrm{s}$. As can be seen from Figure 3, a good agreement 
is obtained between the experimental results and analytical model with eqs 6 and 8 using essentially only three parameters: $\bar{n}, \mu$, and $\alpha$ over a range of AOT concentrations and cell thicknesses. Using $K=\bar{n}^{2} / \bar{n}_{0}{ }^{2}$ with the estimated value $K=2.6$ $\times 10^{-10}$ as explained in the introduction and, the generation rate constant $\beta=2.3 \times 10^{-26} \mathrm{~m}^{3} / \mathrm{s}$ can then be obtained as well. The location of the saturation voltage as well as the level of the generation-limited currents match well.

\section{DISCUSSION}

In this paper steady state currents measured at low and high voltages are used to study the charging dynamics of AOT inverse micelles and to estimate the generation and recombination constants $\alpha$ and $\beta$. The dependence of the steady state current on the applied voltage, micelle concentration, and device thickness allows to identify the mechanism behind the generation of charged inverse micelles. Different mechanisms can be involved in the measured conductivity and currents such as different bulk and surface generation mechanisms. However, in this article we use an $\mathrm{Al}_{2} \mathrm{O}_{3}$ layer to block faradaic currents and to reduce surface generation of AOT inverse micelles, such that we can focus on generation in the bulk.

Our results show specific dependencies of steady state currents upon the applied voltages which can only be explained by a bulk disproportionation mechanism. At low voltages $(V<$ $V_{\text {sat }}$ ), the steady state current increases linearly with the concentration of AOT which is reported in other studies as well. $^{32,44}$ This already indicates that the initial charged inverse micelles have been generated by a disproportionation mechanism. In the case of a dissociation mechanism, we would expect that the current depends on the square root of the AOT concentration. For higher voltages, the steady state current saturates and the saturation level, the generation-limited current, is found to be proportional to the square of the concentration of AOT. This result confirms that the new inverse micelles are generated by a disproportionation mechanism. In the case of dissociation, one would expect that the level of the saturated current depends linearly on the concentration of AOT.

The study of the steady state current to determine the charging dynamics of charged species has its limitations. In some cases surface generation cannot be neglected compared to bulk generation of charged inverse micelles. This happens especially for low concentrations of AOT and for thin devices when insulating coatings on the electrodes cannot completely avoid charge generation at the surface. This phenomenon is more obvious at very high applied voltages $V_{\mathrm{A}}>50 \mathrm{~V}$, where it is observed that the steady state shows a superlinear dependency to the applied voltage. This phenomenon can be explained by a leakage of charge through the electrode coating or by a voltage dependency of $\alpha$ and $\beta$.

In all above measurements, AOT samples are prepared under ambient conditions, and no measures were taken to eliminate humidity in the mixtures. However, we have previously compared the conductivity from our results with other studies in which the water content was minimized using dry and fresh AOT and anhydrous dodecane prepared and introduced in the measurement device under a protective atmosphere. Although the conductivity of AOT solutions increases by adsorbing ambient moisture, our measurements show that the general behavior of AOT inverse micelles is approximately the same. ${ }^{33}$

\section{CONCLUSION}

Detailed measurements of transient currents for different step voltages reveal the generation and recombination dynamics of AOT inverse micelles in dodecane. At voltage steps $V<d^{2} \alpha \sigma /$ $\left(2 e \mu^{2}\right)$ below the saturation voltage, the mixture can be described as a conductivity because generation of charged inverse micelles is sufficiently fast to maintain the steady state concentration of charged inverse micelles. At voltages $V>$ $d^{2} \alpha \sigma /\left(2 z e \mu^{2}\right)$ above the saturation voltage, the current drops in the transit time from its initial current to a generation-limited value that does not depend on the applied voltage. The observations are in good agreement with an analytical model based on bulk disproportionation for the generation of charged AOT inverse micelles with a generation constant $\beta=2.3 \times$ $10^{-26} \mathrm{~m}^{3} / \mathrm{s}$ and a recombination constant of $\alpha=9.0 \times 10^{-17}$ $\mathrm{m}^{3} / \mathrm{s}$.

\section{AUTHOR INFORMATION}

\section{Corresponding Author}

*E-mail: mkarvar@elis.ugent.be.

\section{Notes}

The authors declare no competing financial interest.

\section{REFERENCES}

(1) Comiskey, B.; Albert, J. D.; Yoshizawa, H.; Jacobson, J. An electrophoretic ink for all-printed reflective electronic displays. Nature 1998, 394 (6690), 253-255.

(2) Chen, Y.; Au, J.; Kazlas, P.; Ritenour, A.; Gates, H.; McCreary, M. Flexible active-matrix electronic ink display. Nature 2003, 423 (6936), 136-136.

(3) Bert, T.; De Smet, H.; Beunis, F.; Neyts, K. Complete electrical and optical simulation of electronic paper. Displays 2006, 27 (2), 5055.

(4) Murau, P.; Singer, B. Understanding and Elimination of Some Suspension Instabilities in an Electrophoretic Display. J. Appl. Phys. 1978, 49 (9), 4820-4829.

(5) Kazlas, P. T.; McCreary, M. D. Paperlike microencapsulated electrophoretic materials and displays. MRS Bull. 2002, 27 (11), 894897.

(6) Kim, C. A.; Joung, M. J.; Ahn, S. D.; Kim, G. H.; Kang, S. Y.; You, I. K.; Oh, J.; Myoung, H. J.; Baek, K. H.; Suh, K. S. Microcapsules as an electronic ink to fabricate color electrophoretic displays. Synth. Met. 2005, 151 (3), 181-185.

(7) Hopper, M. A.; Novotny, V. Electrophoretic Display, Its Properties, Model, and Addressing. IEEE Trans. Electron Devices 1979, 26 (8), 1148-1152.

(8) Meng, X. W.; Tang, F. Q.; Peng, B.; Ren, J. Monodisperse Hollow Tricolor Pigment Particles for Electronic Paper. Nanoscale Res. Lett. 2010, 5 (1), 174-179.

(9) Morrison, I. D. Electrical Charges in Nonaqueous Media. Colloids Surf., A 1993, 71 (1), 1-37.

(10) Hao, T. Electrorheological fluids. Adv. Mater. 2001, 13 (24), 1847-+.

(11) Lee, C. H.; Mallinson, R. G. Surfactant Effects in the Emulsion Polymerization of Vinyl-Acetate. J. Appl. Polym. Sci. 1990, 39 (11-12), 2205-2218.

(12) Candau, F.; Leong, Y. S.; Pouyet, G.; Candau, S. Inverse Microemulsion Polymerization of Acrylamide - Characterization of the Water-in-Oil Microemulsions and the Final Microlatexes. J. Colloid Interface Sci. 1984, 101 (1), 167-183.

(13) Hoggan, J. L.; Bae, K.; Kibbey, T. C. G. Non-steady state partitioning of tetrachloroethylene (PCE) dry cleaning surfactants between and water in porous media. J. Contam. Hydrol. 2007, 93 (14), 149-160. 
(14) Wang, S. C.; Yang, H.; Banerjee, S.; Herman, I. P.; Akins, D. L. AOT dispersed single-walled carbon nanotubes for transistor device application. Mater. Lett. 2008, 62 (6-7), 843-845.

(15) Lehr, I. L.; Saidman, S. B.; Schulz, P. C. Iron dissolution in aqueous AOT solution. J. Colloid Interface Sci. 2007, 306 (2), 323327.

(16) Novaira, M.; Moyano, F.; Biasutti, M. A.; Silber, J. J.; Correa, N. M. An example of how to use AOT reverse micelle interfaces to control a photoinduced intramolecular charge-transfer process. Langmuir 2008, 24 (9), 4637-4646.

(17) De, T. K.; Maitra, A. Solution Behavior of Aerosol Ot in Nonpolar-Solvents. Adv. Colloid Interface Sci. 1995, 59, 95-193.

(18) Crespy, D.; Landfester, K. Making dry fertile: a practical tour of non-aqueous emulsions and miniemulsions, their preparation and some applications. Soft Matter 2011, 7 (23), 11054-11064.

(19) Garcia, A. M. F.; Fernandes, M. S. F.; Coutinho, P. J. G. CdSe/ $\mathrm{TiO} 2$ core-shell nanoparticles produced in AOT reverse micelles: Applications in pollutant photodegradation using visible light. Nanoscale Res. Lett. 2011, 6.42610.1186/1556-276X-6-426

(20) Gupta, R. R.; Jain, S. K.; Varshney, M. AOT water-in-oil microemulsions as a penetration enhancer in transdermal drug delivery of 5-fluorouracil. Colloids Surf., B 2005, 41 (1), 25-32.

(21) Meier, W. Kerr effect measurements on a poly(oxyethylene) containing water-in-oil microemulsion. J. Phys. Chem. B 1997, 101 (6), 919-921.

(22) Appel, M.; Spehr, T. L.; Wipf, R.; Stuhn, B. Water-AOTalkylbenzene microemulsions: Influence of alkyl chain length on structure and percolation behavior. J. Colloid Interface Sci. 2012, 376, $140-145$.

(23) Hudson, L. K.; Eastoe, J.; Dowding, P. J. Nanotechnology in action: Overbased nanodetergents as lubricant oil additives. Adv. Colloid Interface Sci. 2006, 123, 425-431.

(24) Kabalnov, A.; Lindman, B.; Olsson, U.; Piculell, L.; Thuresson, $\mathrm{K}$; Wennerstrom, H. Microemulsions in amphiphilic and polymersurfactant systems. Colloid Polym. Sci. 1996, 274 (4), 297-308.

(25) Eicke, H. F. Properties of amphiphilic electrolytes in nonpolar solvents. Pure Appl. Chem. 1981, 53 (7), 1417-1424.

(26) Eicke, H. F.; Borkovec, M.; Das-Gupta, B. Conductivity of water-in-oil microemulsions: a quantitative charge fluctuation model. $J$. Phys. Chem. 1989, 93 (1), 314-317.

(27) Peri, J. B. State of Solution of Aerosol Ot in Nonaqueous Solvents. J. Colloid Interface Sci. 1969, 29 (1), 6-15.

(28) Ekwall, P.; Mandell, L.; Fontell, K. Some Observations on Binary and Ternary Aerosol Ot Systems. J. Colloid Interface Sci. 1970, 33 (2), 215-235.

(29) Zulauf, M.; Eicke, H. F. Inverted Micelles and Microemulsions in the Ternary-System H2o-Aerosol-Ot-Isooctane as Studied by Photon Correlation Spectroscopy. J. Phys. Chem. 1979, 83 (4), 480486.

(30) Eicke, H. F. Aggregation in Surfactant Solutions - Formation and Properties of Micelles and Micro-Emulsions. Pure Appl. Chem. 1980, 52 (5), 1349-1357.

(31) Kotlarchyk, M.; Huang, J. S.; Chen, S. H. Structure of AotReversed Micelles Determined by Small-Angle Neutron-Scattering. J. Phys. Chem. 1985, 89 (20), 4382-4386.

(32) Hsu, M. F.; Dufresne, E. R.; Weitz, D. A. Charge stabilization in nonpolar solvents. Langmuir 2005, 21 (11), 4881-4887.

(33) Karvar, M.; Strubbe, F.; Beunis, F.; Kemp, R.; Smith, A.; Goulding, M.; Neyts, K. Transport of Charged Aerosol OT Inverse Micelles in Nonpolar Liquids. Langmuir 2011, 27 (17), 10386-10391.

(34) Day, R. A.; Robinson, B. H.; Clarke, J. H. R.; Doherty, J. V. Characterization of Water-Containing Reversed Micelles by Viscosity and Dynamic Light-Scattering Methods. J. Chem. Soc., Faraday Trans. 1 1979, 75, 132-139.

(35) Roberts, G. S.; Sanchez, R.; Kemp, R.; Wood, T.; Bartlett, P. Electrostatic charging of nonpolar colloids by reverse micelles. Langmuir 2008, 24 (13), 6530-6541.
(36) Sainis, S. K.; Merrill, J. W.; Dufresne, E. R. Electrostatic Interactions of Colloidal Particles at Vanishing Ionic Strength. Langmuir 2008, 24 (23), 13334-13347.

(37) Parsegian, A. Energy of an Ion Crossing a Low Dielectric Membrane - Solutions to 4 Relevant Electrostatic Problems. Nature 1969, 221 (5183), 844-846.

(38) Strubbe, F.; Verschueren, A. R. M.; Schlangen, L. J. M.; Beunis, F.; Neyts, K. Generation current of charged micelles in nonaqueous liquids: Measurements and simulations. J. Colloid Interface Sci. 2006, 300 (1), 396-403.

(39) Beunis, F.; Strubbe, F.; Marescaux, M.; Neyts, K.; Verschueren, A. R. M. Micellization and adsorption of surfactant in a nonpolar liquid in micrometer scale geometries. Appl. Phys. Lett. 2010, 97 (18).18191210.1063/1.3503968

(40) Karvar, M.; Strubbe, F.; Beunis, F.; Kemp, R.; Smith, N.; Goulding, M.; Neyts, K. Investigation of Various Types of Inverse Micelles in Nonpolar Liquids Using Transient Current Measurements. Langmuir 2014, 30 (41), 12138-12143.

(41) Beunis, F.; Strubbe, F.; Karvar, M.; Drobchak, O.; Brans, T.; Neyts, K. Inverse micelles as charge carriers in nonpolar liquids: Characterization with current measurements. Curr. Opin. Colloid Interface Sci. 2013, 18 (2), 129-136.

(42) Verschueren, A. R. M.; Notten, P. H. L.; Schlangen, L. J. M.; Strubbe, F.; Beunis, F.; Neyts, K. Screening and Separation of Charges in Microscale Devices: Complete Planar Solution of the PoissonBoltzmann Equation. J. Phys. Chem. B 2008, 112 (41), 13038-13050.

(43) Kornilovitch, P.; Jeon, Y. Transient electrophoretic current in a nonpolar solvent. J. Appl. Phys. 2011, 109 (6).06450910.1063/ 1.3554445

(44) Novotny, V. Electrical-Conduction in Surfactant-Water-Nonaqueous Liquid-Systems. J. Electrochem. Soc. 1986, 133 (8), 16291636. 